\title{
In vitro selection and identification of ssDNA aptamers recognizing the Ras protein
}

\author{
ZHI-WEI WANG, HONG-BING WU, ZHI-FU MAO, XIAO-PING HU, \\ HAO ZHANG, ZHI-PENG HU and ZONG-LI REN
}

\author{
Department of Cardiovascular Surgery, Renmin Hospital of Wuhan University, Wuhan, Hubei 430060, P.R. China
}

Received August 28, 2013; Accepted March 4, 2014

DOI: $10.3892 / \mathrm{mmr} .2014 .2337$

\begin{abstract}
The aim of this study was to develop high-affinity single-stranded DNA (ssDNA) aptamers that can selectively recognize the protein Ras and can be used as preventive and therapeutic agents for restenosis occurring after coronary surgery or angioplasty. For this purpose, we used the systematic evolution of ligands by exponential enrichment (SELEX) technique, also known as in vitro selection. Using this technique, ssDNA aptamers recognizing the Ras protein were obtained from a synthesized random ssDNA library in vitro. The binding rate and affinity of each aptamer pool, isolated in successive rounds of selection, were measured using ELISA, and the finally selected aptamer pool was cloned and sequenced. The binding affinities of each aptamer in this pool were measured. Their primary and secondary structures were analyzed using the DNAMAN 5.29 software, and the relationship between these structures and corresponding binding affinities was analyzed. The rate of aptamer pool binding to the Ras protein gradually increased from 2.4 to $34.5 \%$ along the selection process. Optical density (OD) and equilibrium dissociation constant $(\mathrm{Kd})$ measurements showed that OD gradually increased from 0.220 to 1.080 and $\mathrm{Kd}$ decreased from 51.5 to $18.3 \mathrm{nM}$. The 11 th pool of aptamers was selected based on these analyses, and cloning and sequencing of individual aptamers was performed. Secondary structure analysis revealed different conformations, but of a single type: stem-loop. The aptamer Ra1 showed the highest affinity, with a measured OD of 1.213 and an estimated $\mathrm{Kd}$ of $15.3 \mathrm{nM}$. The binding affinity of the aptamer Ra1 to Ras was dose-dependent. In conclusion, high-affinity ssDNA aptamers recognizing the Ras protein have been successfully selected. These aptamers may serve in the future as preventive and/or therapeutic agents for restenosis occurring after coronary surgery or angioplasty.
\end{abstract}

Correspondence to: Dr Hong-Bing Wu, Department of Cardiovascular Surgery, Renmin Hospital of Wuhan University, 238 Jiefang Road, Wuhan, Hubei 430060, P.R. China

E-mail: hbwu2008@163.com

Key words: SELEX, aptamer, Ras protein

\section{Introduction}

Percutaneous coronary intervention and coronary artery bypass grafting are the most important therapeutic modalities for coronary heart disease. However, restenosis occurring after coronary surgery or angioplasty seriously affects the short- and long-term effects of the two treatments. Therefore, restenosis has become an issue that should be addressed. In recent years, a number of different therapies have been developed to treat restenosis, but patients showed low response rates to all of these (1). At present, effective therapies for restenosis are still lacking. Previous studies (2) have shown that the proliferative and migrating abilities of vascular smooth muscle cells (VSMCs) play a very important role in restenosis and are regulated by an intricate signaling pathway network. Ras protein is a critical component of numerous intertwined signal transduction pathways and is a potentially important therapeutic target (3). Ras protein can activate a variety of signaling pathway proteins, such as mitogen-activated protein kinases (MAPKs) and PI3K/ Akt, to induce the proliferation and migration of VSMCs. Therefore, Ras protein may play a crucial role in restenosis. An intervening agent that can effectively inhibit Ras protein activity would be thus highly suitable for the development of preventive and therapeutic treatment for restenosis occurring after coronary surgery or angioplasty. Aptamers have been shown to effectively inhibit the activity of a variety of targets in vitro and in vivo (4). They present a number of advantages over antibodies and antibiotics. Being smaller than antibodies, aptamers show improved cell penetration and blood clearance. Aptamers have been used for pure recognition, inhibition, diagnostic and therapeutic applications (5). To develop a high-affinity, specific inhibitor of the Ras protein, we selected ssDNA aptamers using systematic evolution of ligands by exponential enrichment (SELEX) and analyzed their binding affinities and structures. The high-affinity ssDNA aptamers identified in this study may be used in the future to treat restenosis.

\section{Materials and methods}

Nucleic acids. The oligonucleotide library was synthesized as a single-stranded (ssDNA), 78-bp long DNA fragment, withthefollowingsequence:5'-GGGAGCTCAGAATAAACG 
CTCAA- ${ }_{35}$-TTCGACATGAGGCCCGGATC-3', where $\mathrm{N}_{35}$ is random oligonucleotides based on the equal incorporation of $\mathrm{A}, \mathrm{G}, \mathrm{C}$ and $\mathrm{T}$ at each position. The initial double-stranded DNA (dsDNA) random library was generated by amplification using polymerase chain reaction (PCR) and the primers: forward, 5'-GGGAGCTCAGAATAAACGCTCAA-3' and reverse, 5'-GATCCGGGCCTCATGTCGAA-3'. The oligonucleotide library and the primers were synthesized in vitro by Shanghai Sangon Biological Engineering Co., Ltd. (Shanghai, China).

Ras protein. Recombinant, full-length human wild-type Ras protein (H-Ras) was purchased from Abcam Ltd. (Hong Kong, China).

Optimization of PCR conditions. Symmetric PCR reactions were performed as follows: PCR mixtures contained 1 pmol ssDNA library, 10 pmol forward primer, 10 pmol reverse primer, $10 \mu 1$ of $2 \mathrm{X}$ PCR Master mix $(0.05 \mathrm{U} / \mu 1$ Taq DNA polymerase in reaction buffer, $4 \mathrm{mM} \mathrm{MgCl} 2,0.4 \mathrm{mM}$ dATP, $0.4 \mathrm{mM} \mathrm{dCTP}, 0.4 \mathrm{mM}$ dGTP and $0.4 \mathrm{mM}$ dTTP) and $\mathrm{ds}_{2} \mathrm{O}$, in a total volume of $20 \mu \mathrm{l}$. The mixtures were thermally cycled $5,8,12,16,20,24$ and 28 times through $94^{\circ} \mathrm{C}$ for $3 \mathrm{~min}, 94^{\circ} \mathrm{C}$ for $40 \mathrm{sec}$ and $\mathrm{X}^{\circ} \mathrm{C}$ for $30 \mathrm{sec}$, followed by a 7 -min extension at $72^{\circ} \mathrm{C}$. X was the annealing temperature, which was 53.3, 53.6, 54.4, 55.7, 57.1, 58.6, 60.0, 61.4, 62.9, $64.2,65.0$ and $65.3^{\circ} \mathrm{C}$, respectively. dsDNA PCR products were electrophoresed on a $3 \%$ agarose gel and the appropriate number of cycles and annealing temperature for symmetric PCR were selected based on their electrophoretic profiles. The asymmetric PCR conditions were then optimized. The asymmetric PCR mixtures contained $1 \mu 1 \mathrm{dsDNA}$ template, $100,80,50$ and 30 pmol of forward primer, 1 pmol reverse primer, $10 \mu 12 \mathrm{X}$ PCR Master mix and $\mathrm{ds}_{2} \mathrm{O}$, in a total volume of $20 \mu \mathrm{l}$. The annealing temperature was $60^{\circ} \mathrm{C}$ (defined from optimization of the symmetric PCR reactions) and the number of heating cycles was 30,35 and 40 . The remaining conditions were identical to the symmetric PCR reactions. ssDNA PCR products were electrophoresed on a $3 \%$ agarose gel and electrophoretic profiles were examined in order to choose the most appropriate concentration of the forward primer and the appropriate number of heating cycles for the asymmetric PCR.

In vitro selection. To initiate in vitro selection, $100 \mu \mathrm{l}$ of Ras protein and $100 \mu \mathrm{l}$ sterile $\mathrm{ds}_{2} \mathrm{O}$ (to remove non-specifically bound DNA) were coated in Nunc ${ }^{\mathrm{TM}} 96$-well plates at $4^{\circ} \mathrm{C}$ for $18 \mathrm{~h}$ using $100 \mu \mathrm{l}$ coating buffer $\left(0.05 \mathrm{~mol} / 1 \mathrm{NaHCO}_{3}, \mathrm{pH} 9.6\right)$, and were respectively labelled A and B. After washing, the plates were blocked with $200 \mu 13 \%$ bovine serum albumin (BSA) at $37^{\circ} \mathrm{C}$ for $45 \mathrm{~min}$. After washing, $100 \mu 1$ of the random ssDNA library were first added to $\mathrm{B}$ and incubated with $100 \mu$ l binding buffer $(20 \mathrm{mM}$ HEPES $\mathrm{pH} 7.35,120 \mathrm{mM}$ $\mathrm{NaCl}, 5 \mathrm{mM} \mathrm{KCl}, 1 \mathrm{mM} \mathrm{MgCl}_{2}$ and $1 \mathrm{mM} \mathrm{CaCl}_{2}$ ) at $37^{\circ} \mathrm{C}$ for $45 \mathrm{~min}$. The mixture was then transferred to $\mathrm{A}$ and incubated at $37^{\circ} \mathrm{C}$ for $45 \mathrm{~min}$. A was washed six times with $200 \mu 1$ washing buffer (binding buffer $+0.05 \%$ Tween-20). A total of $200 \mu \mathrm{l}$ eluting buffer $(20 \mathrm{mM}$ Tris-HCl, $4 \mathrm{~mol} / \mathrm{l}$ guanidine thiocyanate and $1 \mathrm{mM}$ DTT, $\mathrm{pH}$ 8.3) was added to A and incubated for $8 \mathrm{~min}$ in a water bath with temperature fixed
Table I. Doses of aptamer pools and Ras proteins in each round of selection.

\begin{tabular}{lrcc}
\hline Round & $\begin{array}{c}\text { ssDNA } \\
(\mathrm{pmol})\end{array}$ & $\begin{array}{c}\text { Ras protein } \\
(\mathrm{pmol})\end{array}$ & ssDNA/Ras \\
\hline 1 & 1,000 & 100 & 10 \\
2 & 500 & 50 & 10 \\
3 & 200 & 20 & 10 \\
4 & 100 & 10 & 10 \\
5 & 100 & 7.5 & 13 \\
6 & 50 & 3.75 & 13 \\
7 & 50 & 2.5 & 20 \\
8 & 30 & 1.5 & 20 \\
9 & 30 & 1.2 & 25 \\
10 & 20 & 0.8 & 25 \\
11 & 20 & 0.6 & 33 \\
\hline
\end{tabular}

at $80^{\circ} \mathrm{C}$. The eluent was collected and DNA was extracted using the UNIQ-10 DNA extraction kit (Shanghai Sangon Biological Engineering Co., Ltd.). The amount of bound ssDNA was quantified on an ultraviolet spectrophotometer. The bound ssDNA was amplified by asymmetric PCR and PCR products were electrophoresed on a $3 \%$ agarose gel. The corresponding bands were cut out and DNA fragments from these were extracted using the EZ-10 Spin Column DNA Gel extraction kit (Bio Basic Inc., Toronto, Canada). The concentration of the extract was determined on an ultraviolet spectrophotometer (UV762; Shanghai Jingke Scientific Instrument Co., Ltd., Shanghai, China). This extract constituted the ssDNA pool for the following round of selection, and was stored at $-20^{\circ} \mathrm{C}$. The selection and amplification steps were reiterated until the binding rates and affinities of the ssDNA pool no longer significantly increased. Table I shows the doses of the aptamer pools and Ras protein in each round of selection.

Binding rates and affinities between aptamer pools and Ras protein. The rates of aptamer pool binding to Ras were measured using the same method as in the in vitro selection, with the following differences in the used quantities: 3 pmol (50 $\mu \mathrm{l})$ of Ras protein or $50 \mu \mathrm{l}$ sterile $\mathrm{dsH}_{2} \mathrm{O}$ were coated, using $50 \mu 1$ of coating buffer; $100 \mu 13 \%$ BSA was used for blocking; 100 pmol $(50 \mu \mathrm{l})$ random ssDNA was added to B and incubated with $50 \mu$ l binding buffer; A was washed with $100 \mu 1$ washing buffer and $100 \mu l$ of eluting buffer was added to $\mathrm{A}$. The binding rate was defined as the proportion of bound ssDNA relative to 100 pmol of total ssDNA.

The affinities of aptamer pool binding to Ras were measured by ELISA. Each pool of aptamers was initially amplified by asymmetric PCR using biotin-labeled primers to obtain biotin-labeled ssDNA. The same procedure as for that used to estimate the binding rates was followed, using the same quantities of each reagent until the step of washing the A solution. Following this step, a total of $100 \mu \mathrm{l}$ streptavidin-peroxidase $(1: 10,000)$ was added to $\mathrm{A}$ and incubated at $37^{\circ} \mathrm{C}$ for $60 \mathrm{~min}$. After washing three times using $100 \mu \mathrm{l}$ 

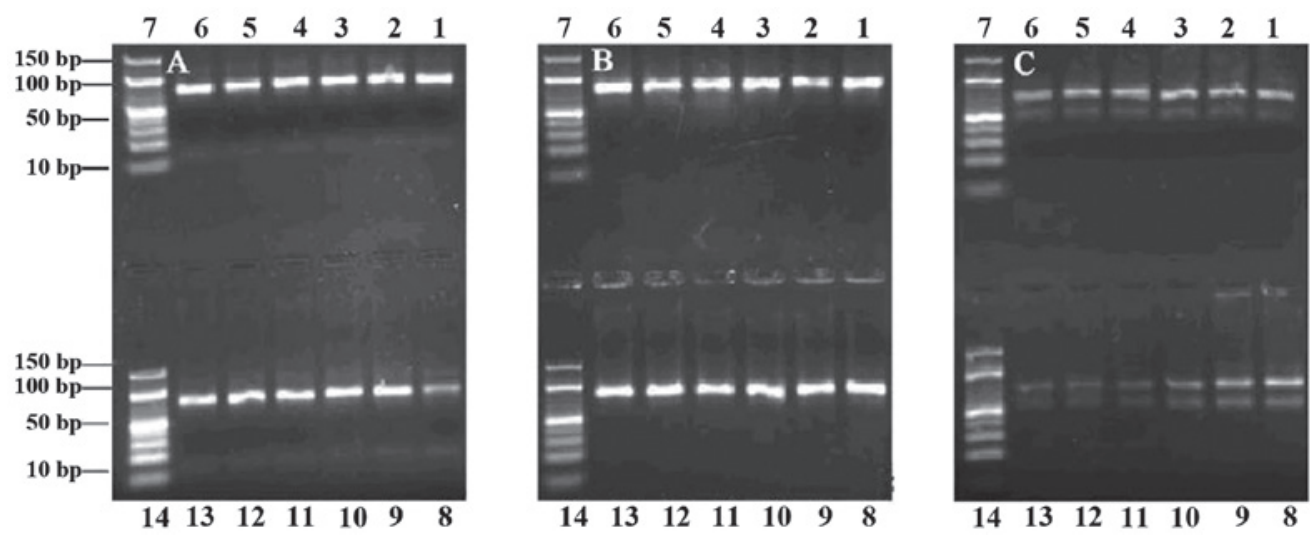

Figure 1. Agarose gel (3\%) electrophoresis analysis of polymerase chain reaction (PCR) products. Number of heating cycles: (A) 5, (B) 24 and (C) 28 . Lanes 1-6 and 8-13, products from PCR performed with annealing temperatures $53.3,53.6,54.4,55.7,57.1,58.6,60.0,61.4,62.9,64.2,65.0$ and $65.3^{\circ} \mathrm{C}$, respectively. Lanes 7 and 14, DNA ladder.

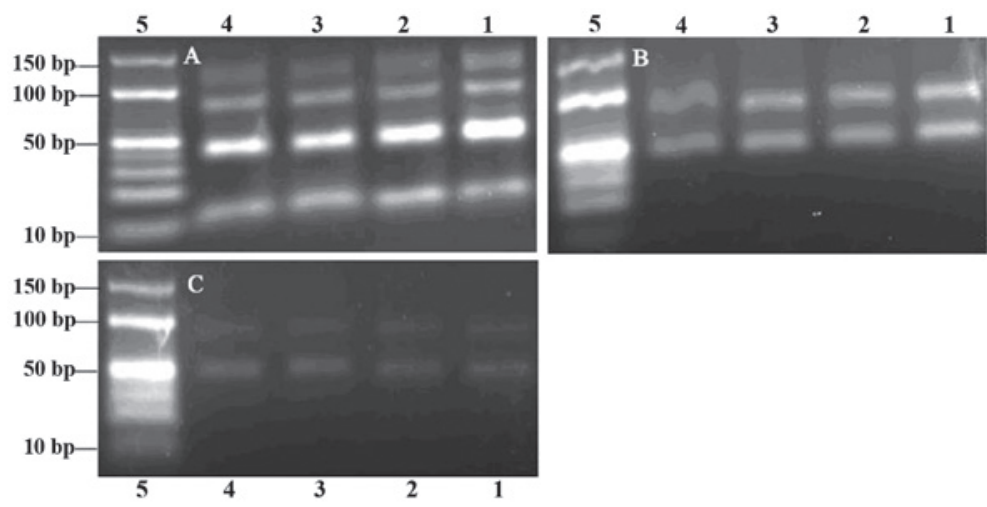

Figure 2. Agarose gel (3\%) electrophoresis analysis of asymmetric polymerase chain reaction (PCR) products. (A) 40, (B) 35 and (C) 30 . Lanes 1-4, the proportion of two primers was 100:1, 80:1, 50:1 and 30:1, respectively. Lane 5, DNA ladder.

washing buffer, A was incubated with the 3,3',5,5'-tetramethylbenzidine (TMB) substrate provided in the ELISA kit (Baosheng, Beijing, China). The optical density (OD) of each sample was then measured at $450 \mathrm{~nm}$ on an ELISA reader (Victor 31420 Multilabel Counter; PerkinElmer, Waltham, MA, USA). The equation $\mathrm{Y}=\mathrm{B}_{\max } \mathrm{x} \mathrm{X} /(\mathrm{Kd}+\mathrm{X})$ was used to calculate the target-antigen binding equilibrium dissociation constant $(\mathrm{Kd})$, where $\mathrm{B}_{\max }$ is the maximum $\mathrm{OD}, \mathrm{Y}$ is the mean $\mathrm{OD}$ and $\mathrm{X}$ is the molar concentration of ssDNA.

Cloning and sequencing of selected aptamers. The 11th pool of aptamers was cloned. Plasmid DNA was isolated from individual clones, purified and analyzed by sequencing. This procedure provided a total of 50 individual aptamers.

Aptamer sequence analysis. The primary and secondary structures of individual aptamers were analyzed based on their sequences using the DNAMAN 5.29 software (Lynnon Corp., Quebec, QC, Canada).

Binding affinity between individual aptamers and Ras protein. Biotin-labeled individual aptamers were synthesized based on their sequences. The binding affinities between individual aptamers and Ras were measured by ELISA, followed by calculation of Kd based on OD values. The same steps described above were followed.

\section{Results}

Optimization of PCR conditions. In the agarose electrophoresis gels, the 78-bp ssDNA fragment migrated between the 40- and 50-bp bands of the dsDNA ladder, and the 78-bp dsDNA near the $80-\mathrm{bp}$ band. PCR products were successfully obtained in symmetric PCR reactions when the number of heating cycles was 5-24 (Fig. 1A and B), while more PCR by-products were observed when this number was $>28$ (Fig. 1C). Visible bands were obtained from the PCR reactions performed with annealing temperatures $53.3-65.3^{\circ} \mathrm{C}$, while when this temperature was $>60^{\circ} \mathrm{C}$, the PCR product quantities began to decrease (Fig. 1C). Since the selection would fail if an extremely high number of PCR by-products were formed, and because we aimed to obtain a high number of specific PCR products, we chose an annealing temperature of $60^{\circ} \mathrm{C}$ and 24 heating cycles as the most appropriate conditions, in order to obtain the maximum number of specific PCR products with a minimal number of PCR by-products formed. Similarly, based on the gel examination of products from the indirect asymmetric PCR (ssDNA was amplified by symmetric PCR to dsDNA and then dsDNA was amplified by asymmetric PCR to ssDNA; Fig. 2), we chose a 100:1 proportion for the forward and reverse primers, and 40 heating cycles. The direct asymmetric PCR amplification (ssDNA was amplified by asymmetric PCR to ssDNA) gave only non-specific by-products and not ssDNA. 
Table II. Ras binding rates (\%), optical density (OD) and equilibrium dissociation constant (Kd; in nM) for aptamer pools from different rounds of selection.

\begin{tabular}{lcccccccccccc}
\hline Round & 1 & 2 & 3 & 4 & 5 & 6 & 7 & 8 & 9 & 10 \\
\hline Rate & 2.4 & 4.0 & 9.5 & 11.5 & 18.7 & 19.1 & 24.4 & 29.4 & 34.3 & 33.9 & 34.5 \\
OD & 0.220 & 0.358 & 0.445 & 0.498 & 0.638 & 0.702 & 0.819 & 0.917 & 1.012 & 0.998 & 1.080 \\
Kd & 51.5 & 48.6 & 47.1 & 47.4 & 39.4 & 39.9 & 32.6 & 24.8 & 19.8 & 19.6 & 18.3 \\
\hline
\end{tabular}

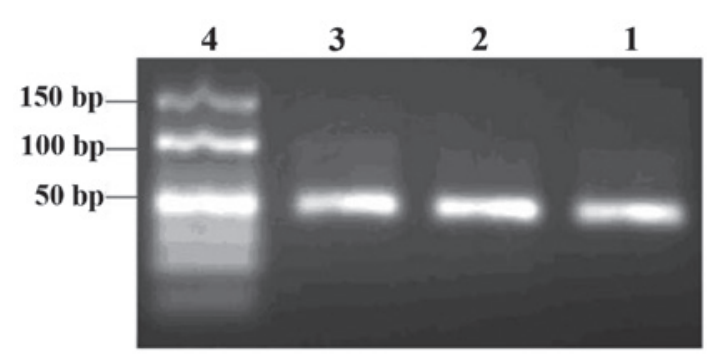

Figure 3. Agarose gel (3\%) electrophoretic analysis of aptamer pools. Lanes 1-3, aptamer pools from the 1st, 5th and 11th round of selection; lane 4, DNA ladder.

In vitro selection. To isolate aptamers that specifically bind to Ras protein, we utilized Ras protein as a selection target. Sterile $\mathrm{ds}_{2} \mathrm{O}$ was used as a negative target control. The random ssDNA library was used to screen ligands that bind to Ras protein. Different pools of aptamers that bind to Ras with high affinity and specificity were successfully obtained after 11 rounds of selection by SELEX. Each pool of aptamers was examined before and after PCR amplification on 3\% agarose gels. The aptamer pools migrated between the 40- and 50-bp bands of the dsDNA ladder, and their PCR products near the 80-bp band of the dsDNA ladder (Figs. 3 and 4).

Binding rates and affinities between the aptamer pools and Ras protein. The binding rates and affinities between the aptamer pools and the Ras protein were calculated. The results showed that the binding rates increased gradually from 2.4 to $34.5 \%$ along the process of the in vitro selection. The binding affinities, as evidenced by measured OD, increased gradually from 0.220 to 1.080 . Kd was calculated based on the equation described in 'Materials and methods'; results are shown in Table II. No further improvement in the binding rates and affinities was observed after 11 rounds of selection. The 11th aptamer pool showed the highest binding rate $(34.5 \%)$ and the highest binding affinity with an OD at 1.080 and a $18.3 \mathrm{nM}$ Kd (Table II).

Cloning and sequencing of selected aptamers. The aptamer pool from the 11th round of selection was cloned. Plasmid DNA was isolated from individual clones, purified and analyzed by sequencing. A total of 50 individual aptamers was thus obtained. The constant regions of these sequences were all identical to the sequence initially designed. There were 45 sequences comprising 35 base pairs in the random region as initially designed, and 5 mutated sequences, among which 4 deletion mutations (one base pair deleted in three aptamers and two base pairs in one) and 1 insertion mutation

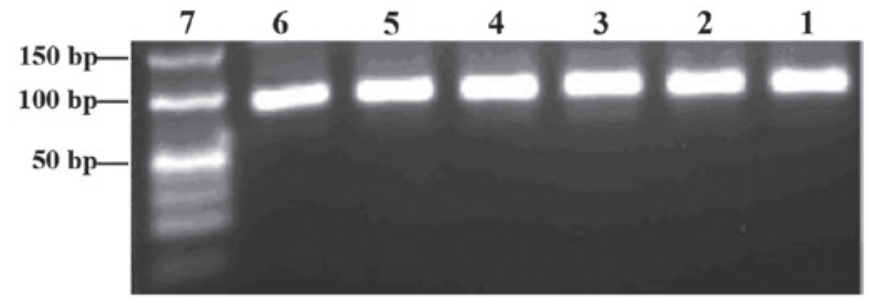

Figure 4. Agarose gel (3\%) electrophoretic analysis of polymerase chain reaction (PCR) products of aptamer pools. Lanes 1-6, aptamer pool PCR products from the 1st, 3rd, 5th, 7th, 9th, and 11th round of selection; lane 7, DNA ladder.

(one base pair). Two of the 50 sequences were identical to each other.

Aptamer sequence analysis. Following rejection of the 5 mutated sequences, the 45 retained sequences were grouped into 10 major families based on their primary structures, analyzed by DNAMAN 5.29 software. Conserved primary structure motifs were observed in aptamers Ra1 to Ra44 grouped in families 1 to 9. Only the sequence of aptamer Ra45, family 10, was not associated with those of the other aptamers (Table III). The secondary structure analysis revealed different conformations among aptamers, but of a single type: stem-loop structure. These stem-loop structures were located at 5'-, 3'-fixed or random sequences, and consisted of 5'-, 3'-fixed, or fixed and random sequences. The most conserved sequences were part of the stem-loop structure with angles.

Binding affinity between individual aptamers and Ras protein. A total of 45 biotin-labeled individual aptamers was synthesized based on their respective sequences. The binding affinities between individual aptamers and Ras were measured by ELISA. The OD of the complexes ranged between 0.459 and 1.213 and their $\mathrm{Kd}$ between 48.3 and $15.3 \mathrm{nM}$ (Table IV). Aptamers Ra1 and $\mathrm{Ra} 2$ had the highest affinity with an OD at 1.213 and a $15.3 \mathrm{nM} \mathrm{Kd}$. Aptamer Ra27 had the lowest affinity with an OD at 0.459 and a $48.3 \mathrm{nM} \mathrm{Kd}$. To explore whether the binding affinity of aptamers to the Ras protein is dose-dependent, a series of mixtures with a fixed concentration of aptamer (100 nM) and increasing concentrations of Ras (0, $0.75,1.50,3.00$ and $6.00 \mathrm{nM}$ ) was prepared for ELISA assay. OD measurements showed that the affinities of Ra1 (Fig. 5) or $\mathrm{Ra} 2$ (data not shown) aptamer binding to the Ras protein are dependent on the aptamer dose.

The data from binding affinity estimations were subsequently mapped to the primary and secondary structures of the corresponding aptamers. We found that most aptamers 
Table III. Sequence of aptamers of different families, grouped based on the presence of common motifs (in bold).

\begin{tabular}{|c|c|}
\hline Aptamer & Random sequence (5'-3') \\
\hline \multicolumn{2}{|l|}{ Family 1} \\
\hline 1 & TCGTCGGATCGACGCGGTTTAGTGAGTGTGCGTGG \\
\hline 2 & TCGTCGGATCGACGCGGTTTAGTGAGTGTGCGTGG \\
\hline 3 & GCTGAAGCAGGGTTGAGTGATAAATGGTGCGTCGG \\
\hline 4 & GGGCAAGCGTCGTGGACAGGAAGAAGTATGTGGTA \\
\hline 5 & GTAAGTGTGTGGTGATTTGGTATATGTAGGCGTCG \\
\hline \multicolumn{2}{|l|}{ Family 2} \\
\hline 6 & GGGGGGGTCGGGAGGTCAGGTTAGGGGGGGTGTTG \\
\hline 7 & GAGGGGCGGAGTATCGGGGGGGGGGGAGGGGGCGG \\
\hline 8 & GAAAGAAGGGGTTGTTGTGAGGGGGGTGGTGGTGG \\
\hline 9 & GCAAAGATTGGGGGGATGATTGAAATAAGCGTGTG \\
\hline 10 & GCGGGGGTGGTTGGGCTGGGGGATGTGGGGTGGTG \\
\hline 11 & TGGGGACGGGGGTATGGGAGGGGGGTGGTGGTGGA \\
\hline 12 & GCTAATGCCGTATGCGCAAGGATGACACACGGGGG \\
\hline 13 & AAGAGTGAGATGATGGATAATGCAAACTGGGGGTG \\
\hline 14 & AGTGCTGCTTGGGGGTTCGTAGTTCGAGTGCTAGG \\
\hline 15 & CGATGGTGTGGTTGGTGGGGGAGTGCGACGCCTGA \\
\hline 16 & TAAGCGCGAGGGGGTATAGGTGTATGTAGCGTGAG \\
\hline \multicolumn{2}{|l|}{ Family 3} \\
\hline 17 & GAGGGATAGGAGTGGTGTTGTTCGTTGTTGCATGG \\
\hline 18 & GTGGAGTATTGGTAGTGATAGAGGGCTTAGGCGGG \\
\hline 19 & GGAGGGGATGACGAGAGCAATCGGCGTGCAAGGGA \\
\hline 20 & GGGAGTTGGCGAATAAGCTTTAGTTGGGAGGGATG \\
\hline 21 & TGGTTGAGGGGAGGCAGAGGGAGGTATAAGGTATG \\
\hline 22 & ACCGGGCGGGTTGCAAGATGGTGAGGGTGCGGGTG \\
\hline 23 & GGAGGGGAAGAAGACGCGAACATGAAAGATGCGTC \\
\hline
\end{tabular}

Family 4

GAGACAGAGCTACGGAGGTGAGATATCAGCATGTG TTGAGCGGGTAATTGGAGTATGTGTGTGTAGGTGG TACTGCAAGAGTGAAAATGTGGTTAGTGAGGTCGG GATGATGTGGGGAGTGGAGTGTAATGGAGAGTGAG GGCGGATGTGAGTTGTTATGAGTTGCAGAGTGGAG GGAGTGTGTTGGGGTGCTATTTGGTTGTATGTGTG

Family 5 30 31 AGCAAAGGAAGACAGGGGAAGTGGTGAAGTTGACG AGAGGATAGTGATGGACGGTGAAGTGCGTGGGTGG AGGGGAAGTGGTGATCGGATAGGACAAAGGAAGTG

Family 6 GAGGTTGGGCTGGAATAAGATTGGTTGTGGGTAGG TGCGAGAAGCGCGGAAAAGGGTAGGCACGGAAGCG GCGTGGTAAGGTCGGCAGCAGGGCGCAGTTGGAGG TGCCGGAACCGGCAGGGAAGGTGTGTGGGTGTGCA GCGGAGGAAGGTAGAGACGATGGCTATAGCGATAG

Family 10 
Table IV. Optical density (OD) and equilibrium dissociation constant $(\mathrm{Kd}$; in $\mathrm{nM})$ ) values of different aptamers.

\begin{tabular}{lcccc}
\hline Aptamer & Ra1 & Ra1-mut & Ra27 & Ra27-mut \\
\hline OD & 1.213 & 0.715 & 0.459 & 0.621 \\
Kd & 15.3 & 27.8 & 48.3 & 34.2 \\
\hline
\end{tabular}

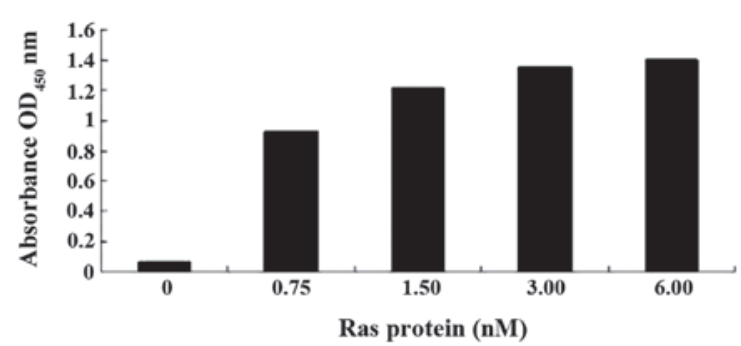

Figure 5. ELISA analysis of binding affinities between aptamer Ra1 and the Ras protein. The concentration of Ra1 was fixed (100 nM), and increasing Ras doses were tested. OD, optical density.

with high binding affinity also had a high number of small stem-loops, while those with low affinity had fewer small stem-loops (Fig. 6). We therefore hypothesized that the terminal loop of a stem-loop structure may be the site of binding to the target Ras protein. To test this hypothesis, aptamers Ra1-mut and Ra27-mut were synthesized, in which two bases in the terminal loop of the stem-loop structure of Ra1 and Ra27 were mutated (Fig. 6C and D). The Ra1- and Ra27-mut affinities of binding to the Ras protein were then measured. The OD of the Ra1-mut aptamer was markedly decreased compared to the original aptamer Ra1, while the opposite trend was observed for aptamer Ra27-mut. Thus, the terminal loop of a stem-loop structure might be the site of binding to the target Ras protein.

\section{Discussion}

Restenosis after coronary surgery or angioplasty remains an issue that needs to be solved (6-8). In-stent restenosis (ISR) occurs in $15-50 \%$ of patients after bare-metal stent implantation and still constitutes one of the most common adverse events (9). Although the use of drug-eluting stents has significantly reduced ISR incidence rates, ISR persists as an unresolved clinical issue, especially for patients presenting highly complex lesions $(10,11)$.

A previous study showed that the proliferation and migration of VSMCs play a very important role in restenosis (12). Ras protein can activate numerous signaling pathway proteins, such as MAPKs and PI3K/Akt, to induce VSMC proliferation and migration (13). Using the SELEX technique, aptamers that bind with high affinity and specificity to a wide range of selected molecular targets can be efficiently isolated from large randomized ssDNA libraries. Aptamers can be highly potent antagonists of specific protein-protein interactions (14). Mutations in the KRAS gene are required for the early occurrence and maintenance of tumorigenesis and are the most frequently found mutations in numerous types of human malignant diseases. Jeong et al (15) identified and characterized an RNA aptamer that specifically binds to the mutant KRAS protein, which could be useful as a ligand for specific therapy and diagnostic applications for cancer types promoted by mutations in this protein. RNA aptamers with affinity for the Ras-binding domain (RBD) of Raf-1 were also isolated from a degenerate pool by in vitro selection. These aptamers efficiently inhibited the interaction of Ras with the Raf-1 RBD, and inhibited Ras-induced Raf-1 activation in a cell-free system (16). The Ras superfamily comprises 50 related proteins bearing a
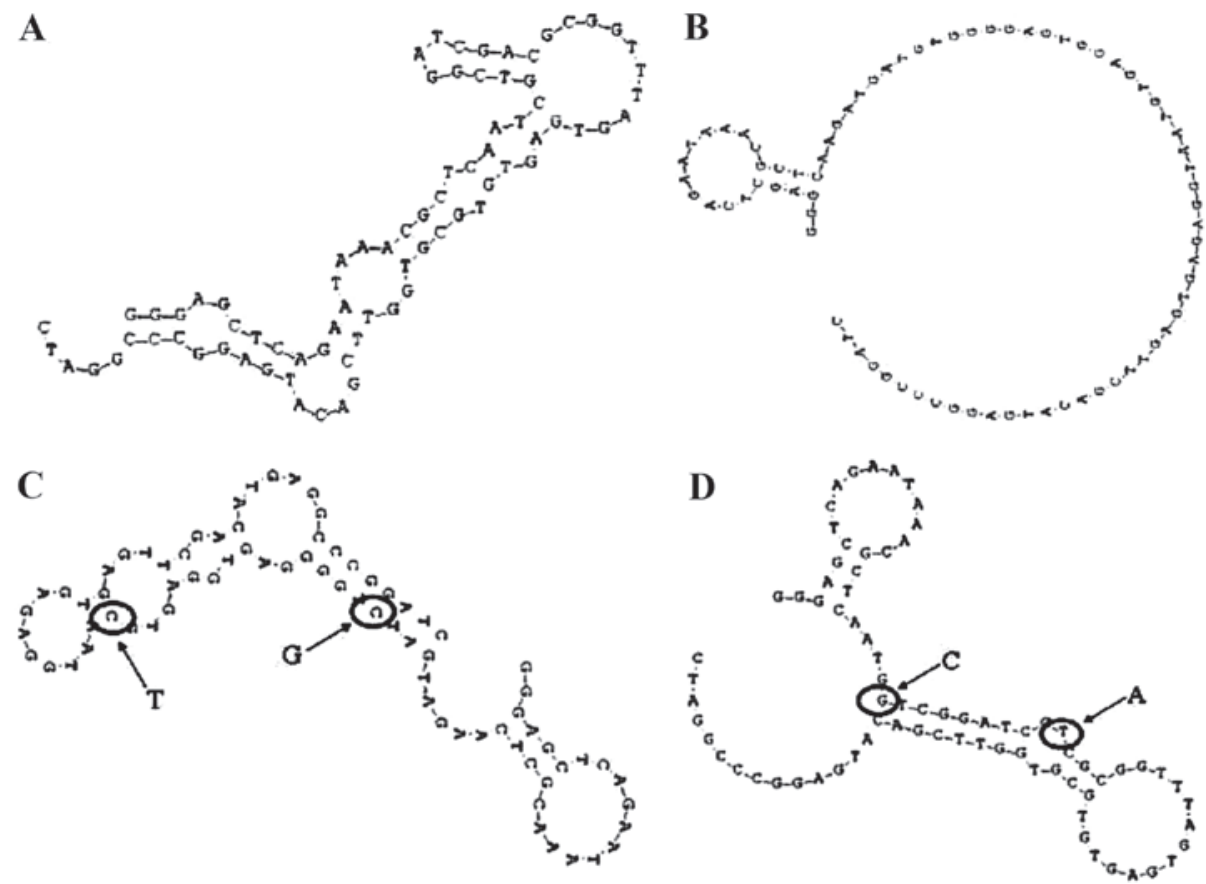

Figure 6. Secondary structure of aptamers (A) Ra1, with a number of small stem-loops, (B) Ra27, with less small stem-loops, (C) Ra1-mut and (D) Ra27-mut. 
GTP-binding domain involved in signal transduction. There are three types of Ras proteins in mammalian cells, H-Ras, $\mathrm{N}$-Ras and K-Ras. The most common of these is H-Ras. Activated H-Ras protein is considered critical for cell proliferation and differentiation (16). Therefore, we attempted to obtain high-affinity ssDNA aptamers recognizing the H-Ras protein by SELEX as a means to treat restenosis occurring after coronary surgery or angioplasty.

In our study, Ras protein was first coated in Nunc 96-well plates, blocked using BSA and then the ssDNA library was added along with binding buffer. The unbound ssDNA was removed and the bound ssDNA, containing the aptamers, was eluted. Bound ssDNA was amplified by asymmetric PCR for the next round of selection. By repeating this process, the bound ssDNA was enriched and the corresponding affinities increased. In the course of the in vitro selection, we set up blank control groups in order to eliminate false positives, i.e., ssDNA non-specifically bound to the plates and/or to BSA. At the beginning of the selection process, since the density of the aptamers was low, the used doses of ssDNA and Ras were high and their proportions were low, in order to isolate more ssDNA with high affinity. At later stages, because the density of the aptamers had increased, the doses of ssDNA and Ras had to be reduced and the rates had to increase. The ssDNA molecules competitively bound to limited concentrations of the Ras protein. Thus, high-affinity ssDNA molecules were enriched and low-affinity ones were removed. Our results showed that the binding rates increased from 2.4 to $34.5 \%$ along the selection process. The binding affinities (based on OD) increased from 0.220 to 1.080 , while Kd decreased from 51.5 to $18.3 \mathrm{nM}$. No further improvement in binding rates and affinities was observed after the 11th round of selection. Sequences from this pool were cloned, and 50 clones were sequenced. The results showed that aptamers with a high affinity to Ras protein were successfully isolated.

ssDNA molecules, which fold into complex secondary and tertiary conformations, including local regions of duplex structures, may be distorted as a result of base mismatches, bulges, pseudoknots and hairpins. The binding affinity of an ssDNA is based on its primary and secondary structures. ssDNA molecules fold, as a function of their primary structure, into complex three-dimensional structures, providing a great diversity of binding specificities. SELEX has been applied to a wide range of targets including growth factors, enzymes and nucleic acids. The aptamer NX-1838, an injectable angiogenesis inhibitor used to treat macular degeneration-induced blindness, showed satisfactory clinical effects (17).

Binding affinities between individual aptamers and Ras were estimated by OD and Kd measurements, with OD ranging between 0.459 and 1.213 and $\mathrm{Kd}$ between 48.3 and $15.3 \mathrm{nM}$. Aptamers Ra1 and Ra2 showed the highest affinity with an OD of 1.213 and a $\mathrm{Kd}$ of $15.3 \mathrm{nM}$. Aptamer Ra27 had the lowest affinity $(\mathrm{OD}=0.459, \mathrm{Kd}=8.3 \mathrm{nM})$. Based on primary structure analyses of these aptamers, conserved primary structure motifs were found to be shared among aptamers Ra1 to Ra44. Only aptamer Ra45 was not related to any of the aptamers. The secondary structure analysis showed that the conformations of the aptamers differed, but that all were of the same type: stem-loop. The stem-loop was located at 5'-, 3'-fixed or random sequences. The stem loops consisted of 5'-, 3'-fixed, or fixed and random sequences. To investigate the intrinsic relationship between the binding affinities and structures, we compared the aptamers with regards to their binding affinities and primary and secondary structures. We found that most of the aptamers with high affinity (e.g., Ra1) had more small stem-loops, while the low-affinity ones, such as Ra27, had fewer. The most conserved sequences were located at stem-loops with angles. A decrease in binding affinity was observed for the aptamer Ra1-mut compared to Ra1, while the OD of Ra27-mut was increased compared to Ra27. The aptamers Ra1-mut, with two mutated bases, and Ra27, had a lower binding affinity compared to Ra1 and Ra27-mut, respectively. The binding affinity of aptamer Ra1 to Ras was dose-dependent. Therefore, these more small stem-loops might be the binding sites of the aptamers to Ras, and conserved motifs within these sequences might play an important role in binding. Li et al (18) also suggested that the stem-loops may be the sites of protein-targeting aptamers, but it was unclear where the conserved sequences located on their sequence. A future study should investigate whether the function of Ras is inhibited by the aptamers identified herein and how this affects VSMC proliferative and migrating ability.

In summary, high-affinity ssDNA aptamers that can bind to the Ras protein have been successfully selected. They could serve as preventive and therapeutic agents for restenosis occurring after coronary surgery or angioplasty in the future.

\section{Acknowledgements}

This study was supported by a grant from the National Natural Science Foundation of China (no. 30872537).

\section{References}

1. Wan S, Shukla N, Yim A, et al: Orally administered penicillamine is a potent inhibitor of neointimal and medial thickening in porcine saphenous vein-carotid artery interposition graft. J Thorac Cardiovasc Surg 133: 494-500, 2007.

2. Liu Q, Lu Z, Zhou H, et al: The mechanical study of vascular endothelial growth factor on the prevention of restenosis after angioplasty. J Tongji Med Univ 21: 195-197, 2001.

3. Jin G, Chieh-His Wu J, Li YS, et al: Effects of active and negative mutants of Ras on rat arterial neointima formation. J Surg Res 94: 124-132, 2000.

4. Cerchia L, Ducongé F, Pestourie C, et al: Neutralizing aptamers from whole-cell SELEX inhibit the RET receptor tyrosine kinase. PLoS Biol 3: e123, 2005.

5. Bunka DH and Stockley PG: Aptamers come of age - at last. Nat Rev Microbiol 4: 588-596, 2006.

6. Lu YG, Chen YM, Li L, Zhao RZ, Fu CH and Yan H: Drug-eluting stents vs. intracoronary brachytherapy for in-stent restenosis: a meta-analysis. Clin Cardiol 34: 344-351, 2011.

7. Reddy MA, Sahar S, Villeneuve LM, Lanting L and Natarajan R: Role of Src tyrosine kinase in the atherogenic effects of the 12/15-lipoxygenase pathway in vascular smooth muscle cells. Arterioscler Thromb Vasc Biol 29: 387-393, 2009.

8. Alfonso F: Interventions for drug-eluting stent restenosis - to cut, or not to cut: is that the question? Circ J 74: 1796-1797, 2010.

9. Kastrati A, Mehilli J, Dirschinger J, Pache J, Ulm K, Schuhlen H, Seyfarth M, Schmitt C, Blasini R, Neumann FJ and Schomig A: Restenosis after coronary placement of various stent types. Am J Cardiol 87: 34-39, 2001. 
10. Liistro F, Fineschi M, Angioli P, Sinicropi G, Falsini G, Gori T, Ducci K, Bravi A and Bolognese L: Effectiveness and safety of sirolimus stent implantation for coronary in-stent restenosis: the TRUE (Tuscany Registry of Sirolimus for Unselected In-Stent Restenosis) registry. J Am Coll Cardiol 48: 270-275, 2006.

11. Aminian A, Kabir T and Eeckhout E: Treatment of drug-eluting stent restenosis: an emerging challenge. Catheter Cardiovasc Interv 74: 108-116, 2009.

12. Kenagy RD, Fukai N, Min SK, Jalikis F, Kohler TR and Clowes AW: Proliferative capacity of vein graft smooth muscle cells and fibroblasts in vitro correlates with graft stenosis. J Vasc Surg 49: 1282-1288, 2009.

13. Tuerk C and Gold L: Systematic evolution of ligands by exponential enrichment: RNA 1igands to bacteriophage T4 DNA polymerase. Science 249: 505-510, 1990.
14. Jeong S, Han SR, Lee YJ, Kim JH and Lee SW: Identification of RNA aptamer specific to mutant KRAS protein. Oligonucleotides 20: 155-161, 2010.

15. Kimoto M, Shirouzu M, Mizutani S, Koide H, Kaziro Y, Hirao I and Yokoyama S: Anti-(Raf-1) RNA aptamers that inhibit Ras-induced Raf-1 activation. Eur J Biochem 269: 697-704, 2002.

16. Wang JX, Tang W, Shi LP, et al: Investigation of the immunosuppressive activity of artemether on T-cell activation and proliferation. Br J Pharmacol 150: 652-661, 2007.

17. Lee JH, Canny MD, De Erkenez A, Krilleke D, Ng YS, Shima DT, Pardi A and Jucker F: A therapeutic aptamer inhibits angiogenesis by specifically targeting the heparin binding domain of VEGF165. Proc Natl Acad Sci USA 102: 18902-18907, 2005.

18. Li ZZ, Han YW, Liu LL, Han YP, Lu Y and Wang CX: In vitro selection of aptamers to gastric cancer cells by SELEX. Biotechnology 19: 42-46, 2009. 